\title{
Monte Carlo simulations of homogeneous upconversion in erbium-doped silica glasses
}

Philipsen, Jacob Lundgreen; Bjarklev, Anders Overgaard

Published in:

I E E E Journal of Quantum Electronics

Link to article, DOI:

$10.1109 / 3.572160$

Publication date:

1997

Document Version

Publisher's PDF, also known as Version of record

Link back to DTU Orbit

Citation (APA):

Philipsen, J. L., \& Bjarklev, A. O. (1997). Monte Carlo simulations of homogeneous upconversion in erbiumdoped silica glasses. I E E E Journal of Quantum Electronics, 33(5), 845-854. https://doi.org/10.1109/3.572160

\section{General rights}

Copyright and moral rights for the publications made accessible in the public portal are retained by the authors and/or other copyright owners and it is a condition of accessing publications that users recognise and abide by the legal requirements associated with these rights.

- Users may download and print one copy of any publication from the public portal for the purpose of private study or research.

- You may not further distribute the material or use it for any profit-making activity or commercial gain

- You may freely distribute the URL identifying the publication in the public portal 


\title{
Monte Carlo Simulations of Homogeneous Upconversion in Erbium-Doped Silica Glasses
}

\author{
Jacob L. Philipsen and Anders Bjarklev, Member, IEEE
}

\begin{abstract}
Quenching of $\mathbf{E r}^{3+}$ ions by homogeneous energytransfer upconversion in high-concentration erbium-doped silica glasses has been theoretically investigated. The results indicate that at $\mathbf{E r}^{3+}$ concentrations of $1.0-2.0 \cdot 10^{26} \mathbf{m}^{-3}$ or below, the kinetic limit of strong migration is not reached, and hence the widely accepted quadratic upconversion model is not generally valid. Nevertheless, the results offer an explanation of the experimental observations of quadratic upconversion. Furthermore, it has been shown that at a given population inversion, the quenching rate depends on the rate of exchange of the excited $\mathrm{Er}^{3+}$ ions by emission and absorption.
\end{abstract}

Index Terms - Concentration quenching, high-concentration erbium-doped amplifiers, upconversion.

\section{INTRODUCTION}

$\mathbf{T}$ HERE HAVE BEEN numerous reports on degradation of performance in high-concentration erbium-doped devices caused by quenching through energy-transfer upconversion between excited $\mathrm{Er}^{3+}$ ions. For erbium-doped fiber amplifiers, a reduction in gain from 18.3 to $6.9 \mathrm{~dB}$ due to quenching is described in [1] for an $\mathrm{Er}^{3+}$ concentration of $6.4 \cdot 10^{25} \mathrm{~m}^{-3}$. In erbium-doped silica waveguides for integrated optics, $\mathrm{Er}^{3+}$ concentrations as high as $1.0-2.0 \cdot 10^{26} \mathrm{~m}^{-3}$ (corresponding to $1.3-2.5 \mathrm{wt} . \% \mathrm{Er}^{3+}$ ) may be necessary to simultaneously ensure sufficient gain and compactness of the components [2], [3], and consequently, quenching is a very serious problem with such devices. For example, a reduction in gain from 37 to $-13 \mathrm{~dB}$ at an $\mathrm{Er}^{3+}$ concentration of $1.15 \cdot 10^{26} \mathrm{~m}^{-3}$ is reported in [2].

The quenching of $\mathrm{Er}^{3+}$ ions through energy-transfer upconversion is usually divided into a contribution from homogeneously distributed $\mathrm{Er}^{3+}$ ions and a contribution from clustered $\mathrm{Er}^{3+}$ ions [4]-[7]. The formation of clusters depends on the fabrication process and the use of co-dopants and may thus to some degree be avoided [8], [9]. In order to estimate the degree of clustering, it is important to be able to interpret measurements on quenching rates in terms of a cluster contribution and an inevitable contribution from homogeneous upconversion. For this purpose, an adequate model for homogeneous upconversion is essential. Furthermore, a model for homogeneous upconversion - together with a model for upconversion in clusters-is a key point in the modeling and design of high-concentration erbium-doped devices [2], [3].

Manuscript received February 5, 1996; revised August 19, 1996.

The authors are with the Center for Broadband Telecommunications, Department of Electromagnetic Systems, Technical University of Denmark, 2800 Lyngby, Denmark.

Publisher Item Identifier S 0018-9197(97)03051-0.
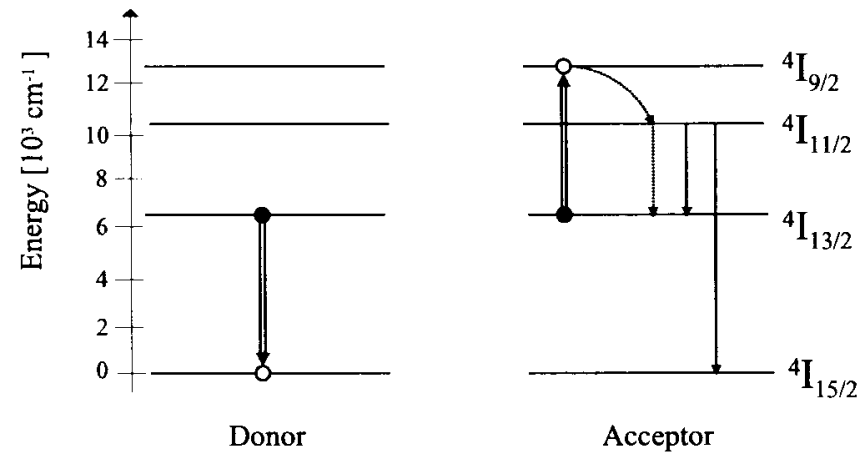

Acceptor

Fig. 1. Energy-transfer upconversion (double arrows) between two $\mathrm{Er}^{3+}$ ions followed by phonon relaxation (dashed arrows) or photon relaxation (solid arrows) for the acceptor ion.

In this paper, we formulate a detailed model for homogeneous upconversion. We derive a system of differential equations for the excitation probabilities of the $\mathrm{Er}^{3+}$ ions and combine this with Monte Carlo simulations of the ion positions. This enables us to perform $a b$ initio calculations of the average quenching rate caused by energy-transfer upconversion. The purpose will be to investigate how the quenching rate depends on various parameters, such as the $\mathrm{Er}^{3+}$ concentration and the population of the upper laser level.

\section{Energy-Transfer Processes Between $E r^{3+}$ IONS}

Fig. 1 illustrates how energy-transfer upconversion between two $\mathrm{Er}^{3+}$ ions, initially excited to the ${ }^{4} I_{13 / 2}$ manifold, leads to the quenching of the donor ion and thus depopulates ${ }^{4} I_{13 / 2}$, which serves as the upper laser level for amplification of light in the 1550-nm wavelength band [2], [4], [10], [11]. The acceptor ion will normally return to the ${ }^{4} I_{13 / 2}$ manifold and only in around $0.1 \%$ of the cases will relax to the ground level ${ }^{4} I_{1 \tilde{\delta} / 2}$ by spontaneous photon emission [4].

Another possible energy-transfer process between two $\mathrm{Er}^{3+}$ ions is illustrated in Fig. 2 [5], [12]. The donor ion relaxes from the ${ }^{4} I_{13 / 2}$ manifold to the ${ }^{4} I_{15 / 2}$ manifold, whereas the acceptor ion makes the reverse transition ${ }^{4} I_{15 / 2} \rightarrow{ }^{4} I_{13 / 2}$. This process does not change the populations of the respective energy levels, but causes diffusion of excitational energy and will thus be referred to as a migration process. Migration will be shown to influence the number of upconversion processes occurring per time and volume unit.

Energy-transfer processes other than the two described above can occur between two interacting $\mathrm{Er}^{3+}$ ions, e.g., cross relaxation between a donor in the ${ }^{4} I_{9 / 2}$ manifold and an acceptor in the ${ }^{4} I_{15 / 2}$ manifold resulting in two $\mathrm{Er}^{3+}$ ions 


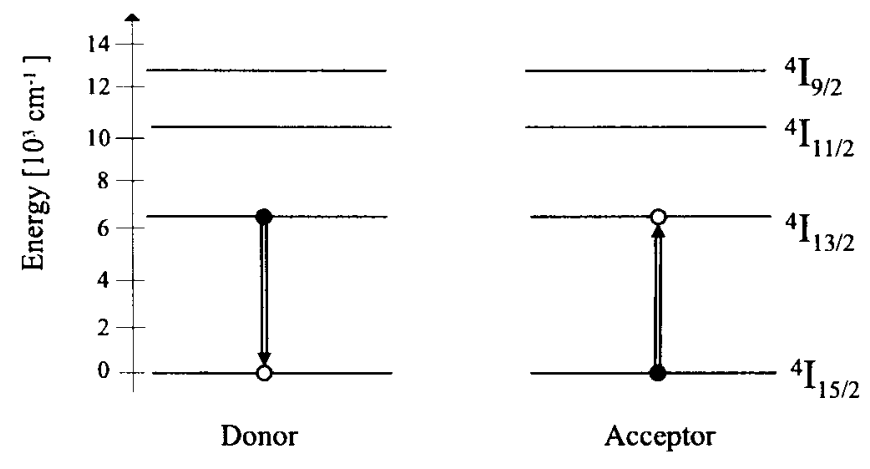

Fig. 2. Migration of excitation from a donor $\mathrm{Er}^{3+}$ ion to an acceptor $\mathrm{Er}^{3+}$ ion.

in the ${ }^{4} I_{13 / 2}$ manifold (the reverse process of upconversion) [3]. However, all these processes involve $\mathrm{Er}^{3+}$ ions initially excited to other energy levels than the manifolds ${ }^{4} I_{15 / 2}$ and ${ }^{4} I_{13 / 2}$. Since the lifetimes of the manifolds ${ }^{4} I_{9 / 2}$ and ${ }^{4} I_{11 / 2}$ are very short $\left(\tau_{9 / 2} \sim 5 \mathrm{~ns}\right.$ and $\tau_{11 / 2} \sim 1-10 \mu \mathrm{s}$, respectively [13]-[15]) compared with the lifetime of the ${ }^{4} I_{13 / 2}$ manifold $\left(\tau_{\mathrm{rad}} \sim 10 \mathrm{~ms}[16]\right)$, the populations of the manifolds ${ }^{4} I_{9 / 2}$ and ${ }^{4} I_{11 / 2}$ are very small, and processes beginning in these energy levels will therefore be neglected. Thus, only the upconversion and migration processes in Figs. 1 and 2 will be considered in this paper.

In the electric dipole-dipole approximation, the probabilities of the described upconversion and migration processes can be expressed in terms of spectral overlap integrals, as shown by Dexter [17]. The probability per time unit of upconversion between two $\mathrm{Er}^{3+}$ ions excited to the ${ }^{4} I_{13 / 2}$ manifold is

$$
P_{\mathbf{u c}}(R)=\frac{12 c}{(2 \pi)^{4} R^{6} n^{2}} \int_{0}^{\infty} \sigma_{\mathrm{em}}(\lambda) \sigma_{\mathrm{ESA}}(\lambda) d \lambda
$$

whereas the probability per time unit of migration from an $\mathrm{Er}^{3+}$ ion excited to the ${ }^{4} I_{13 / 2}$ manifold to an $\mathrm{Er}^{3+}$ ion in the ground level ${ }^{4} I_{15 / 2}$ is

$$
P_{\mathrm{mig}}(R)=\frac{6 c}{(2 \pi)^{4} R^{6} n^{2}} \int_{0}^{\infty} \sigma_{\mathrm{em}}(\lambda) \sigma_{\mathrm{abs}}(\lambda) d \lambda .
$$

In (1) and (2), $R$ is the distance between the nuclei of the two interacting $\mathrm{Er}^{3+}$ ions, $\sigma_{\mathrm{em}}$ and $\sigma_{\mathrm{abs}}$ are the emission and absorption cross sections, respectively, for the ${ }^{4} I_{15 / 2} \leftrightarrow{ }^{4} I_{13 / 2}$ transition, $\sigma_{\mathrm{ESA}}$ is the absorption cross section for the excited state absorption ${ }^{4} I_{13 / 2} \rightarrow{ }^{4} I_{9 / 2}, \lambda$ is the wavelength, $c$ is the speed of light, and $n$ is the index of refraction. The extra factor of 2 in (1) compared to (2) is due to the fact that the upconversion process can occur with either of the two considered $\mathrm{Er}^{3+}$ ions as the donor, respectively the acceptor.

Since the probabilities $P_{\mathbf{u c}}$ and $P_{\mathrm{mig}}$ vary with the ion distance as $R^{-6}$, they can be written

$$
\begin{gathered}
P_{\mathrm{uc}}(R)=\frac{1}{\tau_{\text {rad }}}\left(\frac{R_{\mathrm{uc}}}{R}\right)^{6} \\
P_{\mathrm{mig}}(R)=\frac{1}{\tau_{\text {rad }}}\left(\frac{R_{\mathrm{mig}}}{R}\right)^{6} .
\end{gathered}
$$

The strengths of the upconversion and migration processes are thus described by the two radii $R_{\mathrm{uc}}$ and $R_{\mathrm{mig}}$, indicating the ion distance at which the probability of the respective energytransfer process equals the probability of spontaneous emission for an $\mathrm{Er}^{3+}$ ion excited to ${ }^{4} I_{13 / 2}$.

In [5] and [18], strengths of the upconversion and migration processes have been evaluated for various $\mathrm{Er}^{3+}$-doped silica glasses by calculation of spectral overlap integrals. Using data from these two references, the following values for $R_{\mathrm{uc}}$ are obtained: $0.91 \mathrm{~nm}$ (for Corning1, a barium silicate), $1.0 \mathrm{~nm}$ (for an unspecified aluminoborosilicate), and $1.1 \mathrm{~nm}$ (for Corning2, an aluminoborosilicate). Furthermore, the value $1.8 \mathrm{~nm}$ for $R_{\operatorname{mig}}$ is obtained for the unspecified aluminoborosilicate. Thus, as the typical example, we shall use $R_{\mathrm{uc}} \approx 1.0 \mathrm{~nm}$ and $R_{\text {mig }} \approx 1.8 \mathrm{~nm}$.

\section{Detailed Model For Homogeneous UPCONVERSION}

We begin this section with a brief review of existing theory on energy-transfer upconversion between homogeneously distributed $\mathrm{Er}^{3+}$ ions.

A detailed theory exists-including work by Dexter [17], Eisenthal and Siegel [19], Bŭrshtein [20], Gapontsev and Platonov [21], etc.-describing quenching of a system of excited donors by energy-transfer to a system of acceptors. In discussions on upconversion between $\mathrm{Er}^{3+}$ ions, references are often made to this theory. However, this classical quenching theory deals with donors and acceptors of distinct sorts and is - as will be demonstrated-not adequate for the treatment of self-quenching in $\mathrm{Er}^{3+}$ systems. ${ }^{1}$

In the literature on high-concentration $\mathrm{Er}^{3+}$-doped devices, homogeneous upconversion is often assumed to depend quadratically on the population of the ${ }^{4} I_{13 / 2}$ level, i.e., the number of upconversion processes occurring per time and volume unit is written $g_{\mathbf{u c}}=C_{\mathbf{u c}} n_{2}^{2}$, where $n_{2}$ is the population concentration of the ${ }^{4} I_{13 / 2}$ manifold, and $C_{\mathbf{u c}}$ is an upconversion coefficient [3], [4], [6], [7], [18], [22]. The equivalent average quenching rate experienced by an $\mathrm{Er}^{3+}$ ion in the ${ }^{4} I_{13 / 2}$ manifold is $W_{\mathrm{uc}}=g_{\mathrm{uc}} / n_{2}=C_{\mathrm{uc}} n_{2}$. The quadratic model can be shown theoretically, if migration is assumed sufficiently strong (the kinetic limit) [21] and the model has been supported by some experimental results [4], [23]. Other authors have suggested a cubic model $g_{\mathrm{uc}}=\tilde{C}_{\mathbf{u c}} n_{2}^{3}$ [2], [24].

The present treatment of homogeneous upconversion will be based on the following assumptions.

1) $\mathrm{The}^{3}{ }^{3+}$ ions are assumed randomly distributed with a minimum distance $R_{\min }$ between two neighboring $\mathrm{Er}^{3+}$ ions. This assumption is frequently used within quenching theory [19]-[21], [25] and corresponds to regarding the glass host as a totally disordered material [21]. According to [5], $R_{\min }=0.35 \mathrm{~nm}$ should be applied as the minimum $\mathrm{Er}^{3+}$-ion separation in $\mathrm{Er}^{3+}$ doped silica glasses $(0.35 \mathrm{~nm}$ is the shortest distance between two $\mathrm{Er}^{3+}$ ions in crystalline $\mathrm{Er}_{2} \mathrm{O}_{3}$ ).

2) The electric dipole-dipole approximation and hence (1) and (2) are assumed valid. Although the question of the relative importance of the different contributions to

\footnotetext{
${ }^{1}$ Bŭrshtein [20] treats self-quenching of $\mathrm{Nd}^{3+}$ systems. However, the selfquenching of $\mathrm{Nd}^{3+}$ studied by Bŭrshtein is due to energy-transfer from an excited $\mathrm{Nd}^{3+}$ ion to an unexcited $\mathrm{Nd}^{3+}$ ion and thus differs fundamentally from the self-quenching of $\mathrm{Er}^{3+}$.
} 


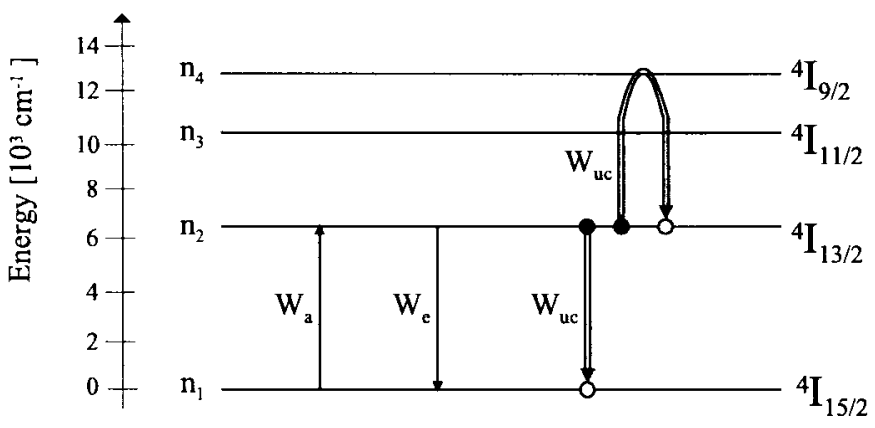

Fig. 3. Two-level model of an $\mathrm{Er}^{3}+$ ion. Single arrows represent radiative transitions, whereas double arrows represent transitions due to energy-transfer upconversion. Rather than showing the physical transitions, the arrows illustrate how the transitions contribute to changing the populations of the energy levels ${ }^{4} I_{15 / 2}$ and ${ }^{4} I_{13 / 2}$, when the lifetimes of the levels ${ }^{4} I_{11 / 2}$ and ${ }^{4} I_{9 / 2}$ are set equal to zero.

the energy-transfer probabilities (electric dipole-dipole, magnetic dipole-dipole, electric dipole-quadrupole, and exchange interaction) seems unresolved [26], it is common within the treatment of energy-transfer between rare-earth ions to assume the electric dipole-dipole interaction to dominate [5], [14], [18], [27].

3 ) Due to the short lifetimes of the manifolds ${ }^{4} I_{11 / 2}$ and ${ }^{4} I_{9 / 2}$, only the ground level ${ }^{4} I_{15 / 2}$ and the upper laser level ${ }^{4} I_{13 / 2}$ are assumed populated. Furthermore, direct relaxation from the ${ }^{4} I_{11 / 2}$ manifold to the ground level ${ }^{4} I_{15 / 2}$ is neglected due to the small branching ratio (c. $0.1 \%$, cf. Section II) for this transition. This implicates that situations with signal light in the 1550$\mathrm{nm}$ wavelength band and pump light in either the 980$\mathrm{nm}$ or the 1480-nm wavelength band can be represented by the common energy level diagram shown in Fig. 3. The total absorption rate $W_{a}$ is the sum of the signal and pump absorption rates, whereas the total emission rate $W_{e}$ is the sum of the rates for spontaneous emission, signal stimulated emission, and-in the case of 1480-nm pump light_-pump stimulated emission.

4) Inhomogeneities of the glass host are neglected, i.e., all $\mathrm{Er}^{3+}$ ions are assumed to have the same radiative properties, and correspondingly the transfer probabilities $P_{\text {uc }}$ and $P_{\text {mig }}$ are only functions of the distance $R$ between the two considered $\mathrm{Er}^{3+}$ ions.

5) Since the purpose is to study upconversion between $\mathrm{Er}^{3+}$ ions, other quenching processes, such as quenching of $\mathrm{Er}^{3+}$ ions through energy-transfer to impurity ions, will be neglected.

Some of the results in this section will, however, be derived using only assumptions 3)-5), i.e., these results will be valid for any ion distribution and any radial dependence of $P_{\mathbf{u c}}$ and $P_{\text {mig. }}$.

We consider a region $\mathcal{V}$ of volume $V$ containing $N_{\mathrm{Er}} \mathrm{Er}^{3+}$ ions at positions $\boldsymbol{r}_{k}, k=1, \cdots, N_{\mathrm{Er}}$, of which $N_{2}$ are excited to the upper laser level ${ }^{4} I_{13 / 2}$, whereas $N_{1}=N_{\mathrm{Er}}-N_{2}$ are in the ground level ${ }^{4} I_{15 / 2}$. The corresponding concentrations are denoted by $n_{\mathrm{Er}}=N_{\mathrm{Er}} / V, n_{1}=N_{1} / V$, and $n_{2}=N_{2} / V$.

If the spatial distribution of the excited $\mathrm{Er}^{3+}$ ions was known, the average upconversion rate $W_{\mathbf{u c}}$ could be determined in a straightforward manner. Let $f^{*}(\boldsymbol{r})$ be the prob- ability distribution for the relative position of two arbitrary excited $\mathrm{Er}^{3+}$ ions. The average upconversion rate would then be given by

$$
W_{\mathbf{u c}}=\left(N_{2}-1\right) \int_{\mathcal{V}} \frac{1}{2} P_{\mathbf{u c}}(|\boldsymbol{r}|) f^{*}(\boldsymbol{r}) d^{3} \boldsymbol{r}
$$

since for an arbitrary excited $\mathrm{Er}^{3+}$ ion the contributions to $W_{\mathbf{u c}}$ from the $N_{2}-1$ other excited $\mathrm{Er}^{3+}$ ions should be summed. However, due to upconversion, the probability of finding a certain $\mathrm{Er}^{3+}$ ion excited to the ${ }^{4} I_{13 / 2}$ manifold will depend on the positions and excitational states of its neighboring $\mathrm{Er}^{3+}$ ions (and will be reduced for $\mathrm{Er}^{3+}$ ions having closely lying excited neighbors). The spatial distribution of excited $\mathrm{Er}^{3+}$ ions will, therefore, differ from the distribution of all $\mathrm{Er}^{3+}$ ions. $^{2}$

Instead, we consider the probability $p_{k}(t)$ of finding the $k$ th $\mathrm{Er}^{3+}$ ion excited at time $t$ together with the joint probability $p_{k \wedge l}(t)$ of finding the $\mathrm{Er}^{3+}$ ions $k$ and $l$ both excited at time $t$. When these probabilities are known, the population inversion can be found as

$$
\frac{n_{2}(t)}{n_{\mathrm{Er}}}=\frac{N_{2}(t)}{N_{\mathrm{Er}}}=\frac{1}{N_{\mathrm{Er}}} \sum_{k=1}^{N_{\mathrm{Er}}} p_{k}(t)
$$

and the number of upconversion processes occurring per time and volume unit is given by

$$
\begin{aligned}
g_{\mathbf{u c}}(t) & =\frac{1}{V} \sum_{k} \sum_{l \neq k} p_{k \wedge l}(t) \frac{1}{2} P_{\mathbf{u c}}\left(\left|\boldsymbol{r}_{k}-\boldsymbol{r}_{l}\right|\right) \\
& =\frac{1}{V} \sum_{k} \sum_{l>k} p_{k \wedge l}(t) P_{\mathbf{u c}}\left(\left|\boldsymbol{r}_{k}-\boldsymbol{r}_{l}\right|\right) .
\end{aligned}
$$

In the Appendix, it is shown that, under assumptions 3)-5), the time development of the single ion probabilities $p_{k}(t)$ is governed by the following system of ordinary differential equations:

$$
\begin{aligned}
\frac{d p_{k}}{d t}= & \sum_{l \neq k}\left[P_{\operatorname{mig}}\left(\left|\boldsymbol{r}_{k}-\boldsymbol{r}_{l}\right|\right)\left(p_{l}-p_{k}\right)-\frac{1}{2} P_{\mathbf{u c}}\left(\left|\boldsymbol{r}_{k}-\boldsymbol{r}_{l}\right|\right) p_{k \wedge l}\right] \\
& -W_{e} p_{k}+W_{a}\left(1-p_{k}\right), \quad k=1, \cdots, N_{\text {Er }}
\end{aligned}
$$

whereas the second-order joint probabilities obey

$$
\begin{aligned}
& \frac{d p_{k \wedge l}}{d t}=\sum_{m \neq k, m \neq l} {\left[P_{\text {mig }}\left(\left|\boldsymbol{r}_{k}-\boldsymbol{r}_{m}\right|\right)\left(p_{l \wedge m}-p_{k \wedge l}\right)\right.} \\
&-\frac{1}{2} P_{\mathbf{u c}}\left(\left|\boldsymbol{r}_{k}-\boldsymbol{r}_{m}\right|\right) p_{k \wedge l \wedge m} \\
&+P_{\text {mig }}\left(\left|\boldsymbol{r}_{l}-\boldsymbol{r}_{m}\right|\right)\left(p_{k \wedge m}-p_{k \wedge l}\right) \\
&\left.-\frac{1}{2} P_{\mathbf{u c}}\left(\left|\boldsymbol{r}_{l}-\boldsymbol{r}_{m}\right|\right) p_{k \wedge l \wedge m}\right] \\
&-2 W_{e} p_{k \wedge l}+W_{a}\left(p_{k}+p_{l}-2 p_{k \wedge l}\right) \\
&-P_{\mathbf{u c}}\left(\left|\boldsymbol{r}_{k}-\boldsymbol{r}_{l}\right|\right) p_{k \wedge l}, \\
& k=1, \cdots, N_{\mathrm{Er}} ; \quad l=k+1, \cdots, N_{\mathrm{Er} .} .
\end{aligned}
$$

In (7), the single ion probabilities $\left\{p_{k}\right\}$ are coupled to the second-order joint probabilities $\left\{p_{k \wedge l}\right\}$, which are then

\footnotetext{
${ }^{2}$ In the classical quenching theory, this important fact was pointed out by Eisenthal and Siegel [19].
} 
coupled to the third-order joint probabilities $\left\{p_{k \wedge l \wedge m}\right\}$ through (8). This pattern will continue if differential equations for higher order joint probabilities are considered. For the numerical calculations in Section IV, it will thus be necessary to truncate the equation system at a certain order and introduce an approximation for the joint probabilities of one order higher. Numerical investigations will then show that two is a suitable order of truncation.

Since it is known from the above mentioned classical quenching theory that quenching rates will in general not be the same under dynamic and stationary conditions [19], and since stationary conditions are the more relevant to modeling of erbium-doped devices, we shall concentrate on the steadystate solution to (7) and (8).

In the following, analytical results will be derived for some special cases. First, we consider the simple case of no upconversion $\left(P_{\mathbf{u c}}=0\right)$. The steady-state solution to (7) and (8) is then found to be

$$
\begin{aligned}
p_{k} & =\frac{W_{a}}{W_{a}+W_{e}}=\frac{n_{2}}{n_{\mathrm{Er}}} \\
p_{k \wedge l} & =\frac{W_{a}\left(p_{k}+p_{l}\right)}{2\left(W_{a}+W_{e}\right)}=\left(\frac{n_{2}}{n_{\mathrm{Er}}}\right)^{2}
\end{aligned}
$$

unaffected by migration. All $\mathrm{Er}^{3+}$ ions are seen to have the same probability of excitation, which thus equals the population inversion. Since $p_{k \wedge l}=p_{k} p_{l}$, the ions are uncorrelated two by two.

When upconversion is included, it is generally not possible to write down the steady-state solution of (7) and (8). To illustrate the effect of upconversion, we therefore consider two closely spaced $\mathrm{Er}^{3+}$ ions $k$ and $l$ that are isolated from all other $\mathrm{Er}^{3+}$ ions. We then find

$$
p_{k}=p_{l}=\frac{W_{a}}{W_{a}+W_{e}}-\frac{P_{\mathrm{uc}}\left(\left|\boldsymbol{r}_{k}-\boldsymbol{r}_{l}\right|\right) p_{k \wedge l}}{2\left(W_{a}+W_{e}\right)} .
$$

Comparison of (10) and (9) shows that upconversion as expected reduces the steady-state probabilities of finding the respective ions excited to the ${ }^{4} I_{13 / 2}$ manifold. Furthermore, it can be shown that

$$
p_{k \wedge l}=\frac{\left(W_{a}+W_{e}\right)^{2}}{\left(W_{a}+W_{e}\right)^{2}+A} p_{k} p_{l}
$$

where

$$
\begin{aligned}
A= & \frac{\frac{1}{4} P_{\mathbf{u c}}^{2}\left(\left|\boldsymbol{r}_{k}-\boldsymbol{r}_{l}\right|\right) W_{a}^{2}}{\left(W_{a}+W_{e}\right)^{2}+\frac{1}{2} P_{\mathbf{u c}}\left(\left|\boldsymbol{r}_{k}-\boldsymbol{r}_{l}\right|\right)\left(2 W_{a}+W_{e}\right)} \\
& +\frac{1}{2} P_{\mathbf{u c}}\left(\left|\boldsymbol{r}_{k}-\boldsymbol{r}_{l}\right|\right) W_{e}>0 .
\end{aligned}
$$

Equations (11) and (12) implicate $p_{k \wedge l}<p_{k} p_{l}$. Thus, upconversion introduces negative correlation between the $\mathrm{Er}^{3+}$ ions: if a given $\mathrm{Er}^{3+}$ ion $k$ is known to be excited, this reduces the probability of finding a near $\mathrm{Er}^{3+}$ ion $l$ excited.

Next, we return to the full system of $\mathrm{Er}^{3+}$ ions and consider the kinetic limit by letting the migration process become infinitely strong, i.e., $P_{\mathrm{mig}}(R) \rightarrow \infty$ for any fixed $R$. From (7) and (8), it then follows that under steady-state conditions all $\mathrm{Er}^{3+}$ ions have the same probability of excitation (which thus equals the population inversion), and that all second-order joint probabilities have a common value. Since ions lying sufficiently far apart must be uncorrelated, this common value for the second-order joint probabilities must equal the square of the population inversion. Thus, in the kinetic limit, we have for all $(k, l)$

$$
p_{k}=\frac{n_{2}}{n_{\mathrm{Er}}}, \quad p_{k \wedge l}=\left(\frac{n_{2}}{n_{\mathrm{Er}}}\right)^{2} .
$$

Although (13) resembles (9), the population inversion will still be reduced compared to (9). In the kinetic limit of strong migration, upconversion affects (reduces) the number of excited $\mathrm{Er}^{3+}$ ions, but does not affect the distribution of excited $\mathrm{Er}^{3+}$ ions.

By inserting (13) into (6), a general expression for the number of upconversion processes occurring per time and volume unit in the kinetic limit is reached:

$$
g_{\mathrm{uc}}^{\mathrm{kin}}=\left(\frac{n_{2}}{n_{\mathrm{Fr}}}\right)^{2} \frac{1}{V} \sum_{k} \sum_{l>k} P_{\mathrm{uc}}\left(\left|\boldsymbol{r}_{k}-\boldsymbol{r}_{l}\right|\right)=C_{\mathrm{uc}}^{\mathrm{kin}} n_{2}^{2} .
$$

From (14), it is seen that in the kinetic limit upconversion depends quadratically on the population inversion for any ion distribution and any radial dependance of the upconversion probability $P_{\mathrm{uc}}$. If furthermore we use the assumption of dipole-dipole interaction, (14) can be rewritten as

$$
g_{\mathrm{uc}}^{\mathrm{kin}}=\left(\frac{n_{2}}{n_{\mathrm{Er}}}\right)^{2} \frac{R_{\mathrm{uc}}^{6}}{V \tau_{\mathrm{rad}}} \sum_{k} \sum_{l>k} \frac{1}{\left|\boldsymbol{r}_{k}-\boldsymbol{r}_{l}\right|^{6}} .
$$

By finally averaging (15) over the assumed random ion distribution, we find the expression

$$
g_{\mathrm{uc}}^{\mathrm{kin}}=n_{2}^{2} \frac{2 \pi R_{\mathrm{uc}}^{6}}{3 \tau_{\mathrm{rad}} R_{\min }^{3}}
$$

which corresponds ${ }^{3}$ to the expression for the kinetic limit in the classical quenching theory [21]. The applicability of the classical quenching theory in the kinetic limit is expectable, since the strong migration erases the special features of selfquenching.

If migration is not strong enough for the kinetic limit to be valid, the steady-state excitation probabilities for $\mathrm{Er}^{3+}$ ions with many close $\mathrm{Er}^{3+}$ neighbors are expected to be lower than average ( $=$ the population inversion), and furthermore negative correlation will be present between the $\mathrm{Er}^{3+}$ ions, i.e., $p_{k \wedge l}<p_{k} p_{l}$. Both these effects tend to reduce the extent of upconversion compared with the kinetic limit, i.e., $g_{\mathrm{uc}}<g_{\mathrm{uc}}^{\text {kin }}$. In other words, under steady-state conditions, upconversion affects the distribution of excited $\mathrm{Er}^{3+}$ ions in a manner that reduces upconversion.

As a final topic before turning to numerical calculations, we consider upconversion at low population inversions $n_{2} / n_{\mathrm{Er}} \ll$ 1. For an arbitrary $\mathrm{Er}^{3+}$ ion $k$, the probability of being quenched by upconversion with another $\mathrm{Er}^{3+}$ ion will vanish as $n_{2} / n_{\mathrm{Er}} \rightarrow 0$. At sufficiently low population inversions, the single-ion excitation probabilities will, therefore, remain unperturbed by upconversion:

$$
p_{k} \approx \frac{n_{2}}{n_{\mathrm{Er}}} \approx \frac{W_{a}}{W_{a}+W_{e}} \approx \frac{W_{a}}{W_{e}}, \quad \frac{n_{2}}{n_{\mathrm{Er}}} \ll 1 .
$$

${ }^{3}$ Equation (16) lacks a factor of two compared with [21], because $P_{\mathrm{uc}}=$ $R_{\mathrm{uc}}^{6} /\left(\tau_{\mathrm{rad}} R^{6}\right)$ in the present text has been defined to be the total probability per time unit of two excited $\mathrm{Er}^{3+}$ ions performing an upconversion process with either of the two ions as the donor. 
The second-order joint probabilities $\left\{p_{k \wedge l}\right\}$ will, however, not all be equal to $\left(n_{2} / n_{\mathrm{Er}}\right)^{2}$, since the negative correlation effect will be present at arbitrarily low population inversion. Instead, the second-order joint probabilities should be found as the steady-state solution to (8). By inserting $d / d t=0, p_{k} \approx p_{l} \approx$ $n_{2} / n_{\mathrm{Er}}$, and $W_{a} \approx W_{e} n_{2} / n_{\mathrm{Er}}$ into (8) and by omitting terms of the order of $\left(n_{2} / n_{\mathrm{Er}}\right)^{3},(8)$ can be rewritten as

$$
\begin{gathered}
-\sum_{m \neq k, m \neq l}\left(P_{\mathrm{mig}}\left(\left|\boldsymbol{r}_{k}-\boldsymbol{r}_{m}\right|\right) p_{l \wedge m}+P_{\mathrm{mig}}\left(\left|\boldsymbol{r}_{l}-\boldsymbol{r}_{m}\right|\right) p_{k \wedge m}\right) \\
+\left[\sum_{m \neq k, m \neq l}\left(P_{\mathrm{mig}}\left(\left|\boldsymbol{r}_{k}-\boldsymbol{r}_{m}\right|\right)+P_{\mathrm{mig}}\left(\left|\boldsymbol{r}_{l}-\boldsymbol{r}_{m}\right|\right)\right)\right. \\
\left.+2 W_{e}+P_{\mathrm{uc}}\left(\left|\boldsymbol{r}_{k}-\boldsymbol{r}_{l}\right|\right)\right] p_{k \wedge l}=2 W_{e}\left(\frac{n_{2}}{n_{\mathrm{Er}}}\right)^{2} \\
k=1, \cdots, N_{\mathrm{Er}} ; \quad l=k+1, \cdots, N_{\mathrm{Fr}} .
\end{gathered}
$$

Since the coefficients on the left-hand side of the linear equational system (18) are independent of the population inversion, and the right-hand side is proportional to the square of the population inversion, the solution must also be proportional to the square of the population inversion, i.e.,

$$
p_{k \wedge l}=p_{k \wedge l}^{(0)}\left(\frac{n_{2}}{n_{\mathrm{Er}}}\right)^{2}
$$

where the normalized probabilities $\left\{p_{k \wedge l}^{(0)}\right\}$ are independent of the population inversion. Insertion into (6) now yields

$$
g_{\mathrm{uc}}=\left(\frac{n_{2}}{n_{\mathrm{Er}}}\right)^{2} \frac{1}{V} \sum_{k} \sum_{l>k} p_{k \wedge l}^{(0)} P_{\mathrm{uc}}\left(\left|\boldsymbol{r}_{k}-\boldsymbol{r}_{l}\right|\right)=C_{\mathrm{uc}}^{(0)} n_{2}^{2} .
$$

We have thus shown that upconversion always depends quadratically on the excited state population at low population inversions.

In the special case of static upconversion, i.e., upconversion under conditions where the influence of migration can be neglected (as we shall see in Section IV, the importance of migration depends on the host glass as well as on the $\mathrm{Er}^{3+}$ concentration), (18) can be solved directly:

$$
p_{k \wedge l}=\left(\frac{n_{2}}{n_{\mathrm{Er}}}\right)^{2} \frac{2 W_{e}}{2 W_{e}+P_{\mathrm{uc}}\left(\left|\boldsymbol{r}_{k}-\boldsymbol{r}_{l}\right|\right)} .
$$

By inserting (21) into (6), by using the assumption of dipole-dipole interaction, and by averaging over the assumed random ion distribution, we find

$$
\begin{aligned}
g_{\mathbf{u c}}^{\text {stat }}= & C_{\mathbf{u c}}^{(0)} n_{2}^{2}=n_{2}^{2} \frac{2 \pi R_{\mathbf{u c}}^{3}}{3 \tau_{\mathrm{rad}}} \sqrt{2 W_{e} \tau_{\mathrm{rad}}} \\
& \times\left[\frac{\pi}{2}-\operatorname{Arctan}\left(\frac{R_{\mathrm{min}}^{3}}{R_{\mathbf{u c}}^{3}} \sqrt{2 W_{e} \tau_{\mathrm{rad}}}\right)\right], \quad \frac{n_{2}}{n_{\mathrm{Fr}}} \ll 1 .
\end{aligned}
$$

We have thus derived an analytical expression describing static upconversion at low population inversions. An interesting feature of (22) is the dependence on the total emission rate $W_{e}$. Letting $W_{e} \rightarrow \infty$ in (22) yields

$$
g_{\mathbf{u c}}^{\text {stat }}\left(W_{e}=\infty\right)=n_{2}^{2} \frac{2 \pi R_{\mathbf{u c}}^{6}}{3 \tau_{\text {rad }} R_{\text {min }}^{3}}
$$

identical to the expression (16) for upconversion in the kinetic limit. The importance of $W_{e}$ is physically interpreted as follows: increasing $W_{e}$ for a fixed population inversion corresponds to increasing both the emission rate $W_{e}$ and the absorption rate $W_{a}$, which leads to an increased exchange of ions in the ${ }^{4} I_{13 / 2}$ manifold. Since emission and absorption happen to random $\mathrm{Er}^{3+}$ ions, the increased exchange will tend to even out the differences (caused by upconversion) in excitation probabilities and will tend to break down the negative correlation between the $\mathrm{Er}^{3+}$ ions (also caused by upconversion). Therefore, the exchange of excited ions by emission and absorption has an effect similar to that of migration. This phenomenon has-to our knowledge-not been described before.

\section{Monte Carlo Simulations}

The description of a system of interacting $\mathrm{Er}^{3+}$ ions contains two stochastic phenomena: the positions and the behavior of the ions, where the latter refers to all transitions between energy levels. This leads to two different principles of numerical simulations: a fully stochastic and a partly stochastic approach. Both methods apply a random number generator for distributing the $\mathrm{Er}^{3+}$ ions in a cubic region $\mathcal{V}$ according to the assumed random ion distribution with a minimum ion separation of $R_{\min }=0.35 \mathrm{~nm}$ (see Section III, assumption 1). In the fully stochastic approach, the random number generator is also used for simulating the behavior of the $\mathrm{Er}^{3+}$ ions, whereas the partly stochastic method applies a numerically found steady-state solution to (7) and (8) for describing the behavior of the $\mathrm{Er}^{3+}$ ions. In both methods, energy-transfer processes are only considered between $\mathrm{Er}^{3+}$ ions separated by a distance smaller than a certain $R_{\max }$.

The partly stochastic method is implemented with two different orders of truncation. In the first-order version, only (7) for the single-ion excitation probabilities is solved, using the approximation

$$
p_{k \wedge l} \approx p_{k} p_{l}
$$

which corresponds to neglecting all negative correlation between the $\mathrm{Er}^{3+}$ ions. In the second-order version, both (7) and (8) are solved, using approximations for the third-order joint probabilities of the form (")" denotes conditioned probability)

$$
\begin{aligned}
p_{k \wedge l \wedge m} & =p_{k \wedge l} p_{m \mid(k \wedge l)} \\
& \approx p_{k \wedge l} p_{m \mid k}=\frac{p_{k l \wedge} p_{k \wedge m}}{p_{k}} .
\end{aligned}
$$

The fully stochastic method, which is characterized by long computation times and noisy results, will only be used for verification of the partly stochastic approach. For this purpose, we consider in Fig. 4 the upconversion rate $W_{\mathbf{u c}}=g_{\mathbf{u c}} / n_{2}$ as a function of the population inversion at an $\mathrm{Er}^{3+}$ concentration of $6.0 \cdot 10^{25} \mathrm{~m}^{-3}$. The three curves in the figure are all based on the same system of $500 \mathrm{Er}^{3+}$ ions, but are calculated 


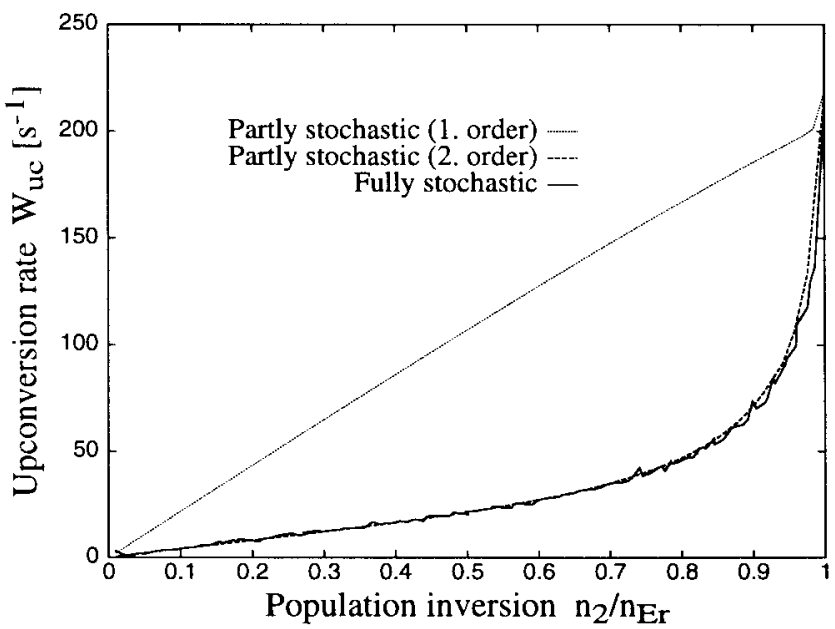

Fig. 4. Comparison of upconversion rates obtained by fully and partly stochastic approaches. The results have been calculated using $n_{\mathrm{Er}}=6.0$ $\cdot 10^{25} \mathrm{~m}^{-3}, W_{e}=1 / \tau_{\mathrm{rad}}=1 /(10 \mathrm{~ms}), R_{\mathrm{uc}}=1.0 \mathrm{~nm}, R_{\mathrm{mig}}=$ $1.8 \mathrm{~nm}, R_{\min }=0.35 \mathrm{~nm}, R_{\max }=3 R_{\mathrm{uc}}$, and by considering a system of $N_{\mathrm{Er}}=500 \mathrm{Er}^{3}+$ ions.

by use of different methods for simulation of the behavior of the ions. The first-order, partly stochastic method is seen to disagree with the fully stochastic method, whereas an acceptable accordance is observed between the second-order, partly stochastic and the fully stochastic method.

According to Fig. 4, the first-order, partly stochastic method wrongly predicts that the system of $\mathrm{Er}^{3+}$ ions is in the kinetic limit. ${ }^{4}$ The interpretation of this is that migration in the present example is sufficiently strong for evening out the differences in single-ion excitation probabilities. However, migration is not strong enough for removing the negative correlation between the $\mathrm{Er}^{3+}$ ions, and the actual upconversion rate therefore-as shown by the two other curves in Fig. 4-will be essentially lower than for the case of the kinetic limit.

The failure of the first-order, partly stochastic method shows the necessity of including the negative correlation effect in the description of interacting $\mathrm{Er}^{3+}$ ions. As mentioned in Section II, the classical quenching theory treats interaction between donors and acceptors of distinct sorts. In such systems, a negative correlation effect does not occur, and the behavior of the ions is, therefore, described in terms of single-ion excitation probabilities [19], [20]. Thus, the results of the classical quenching theory can not be expected to be valid for self-quenching of $\mathrm{Er}^{3+}$.

Since two is thus the appropiate order of truncation, we shall use the second-order partly stochastic method for the following examinations. Due to limitations in computer capacity, systems of only 500 or $1000 \mathrm{Er}^{3+}$ ions will be considered. Sufficient statistical averaging will be ensured by calculation of averages over several systems of $\mathrm{Er}^{3+}$ ions.

We begin by considering static upconversion, i.e., migration is neglected. For an $\mathrm{Er}^{3+}$ concentration of $n_{\mathrm{Fr}}=1.0$. $10^{26} \mathrm{~m}^{-3}$, Fig. 5 shows the upconversion rate $W_{\mathrm{uc}}$ as a function of the population inversion $n_{2} / n_{\mathrm{Fr}}$ for different

\footnotetext{
${ }^{4}$ Notice that when upconversion rate versus population inversion is shown, quadratic upconversion $g_{\mathrm{uc}}=C_{\mathrm{uc}} n_{2}^{2}$ will be represented by a straight line $W_{\mathrm{uc}}=C_{\mathrm{uc}} n_{2}$.
}

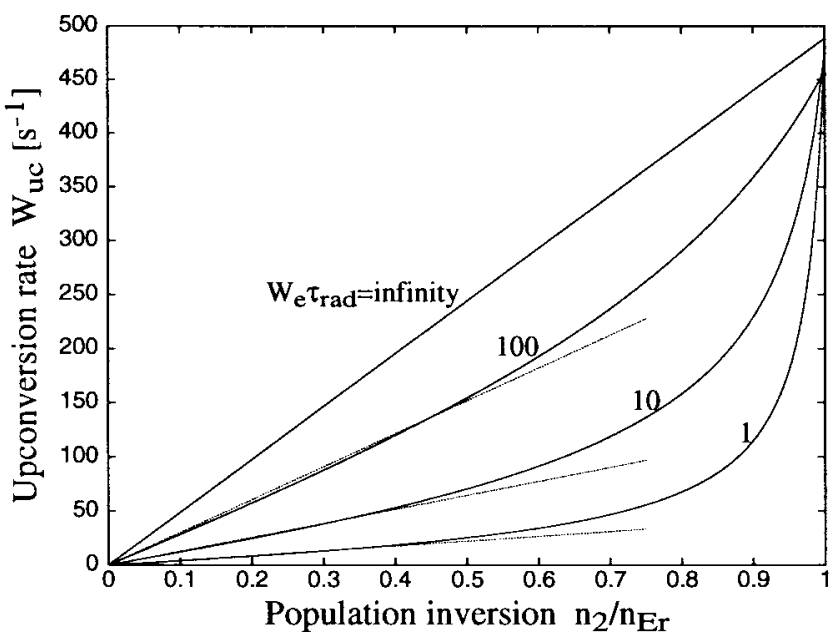

Fig. 5. Upconversion rate versus population inversion for static upconversion at an $\mathrm{Er}^{3+}$ concentration of $n_{\mathrm{Er}}=1.0 \cdot 10^{26} \mathrm{~m}^{-3}$ for different values of the total emission rate $W_{e}$. Other parameter values are $R_{\mathrm{uc}}=1.0 \mathrm{~nm}, R_{\max }=2 R_{\mathrm{uc}}$, and $\tau_{\mathrm{rad}}=10 \mathrm{~ms}$. The curves represent averages over 100 systems, each containing $1000 \mathrm{Er}^{3+}$ ions.

values of the total emission rate $W_{e}{ }^{5}$ The tangents at zero represent the expression (22) for static upconversion at low population inversion and are seen to agree with the simulation results. The straight line representing the case $W_{e} \tau_{\text {rad }}=\infty$ has been drawn by use of the expression (16) for upconversion in the kinetic limit.

The parameter $W_{e} \tau_{\text {rad }}$ is the ratio of the total number of radiative transitions ${ }^{4} I_{13 / 2} \rightarrow{ }^{4} I_{15 / 2}$ (stimulated + spontaneous) to the number of spontaneous radiative transitions ${ }^{4} I_{13 / 2} \rightarrow{ }^{4} I_{15 / 2}$. By analyzing data from [28] for a fiber power amplifier, values above 100 are obtained for $W_{e} \tau_{\mathrm{rad}}$ in the center of the fiber core at the output end of the amplifier. Values of $W_{e} \tau_{\text {rad }}$ ranging between 1 and 10 are expected to be typical for waveguide amplifiers [29]. According to Fig. 5, the parameter $W_{e} \tau_{\text {rad }}$ has great importance for the upconversion rate. For example, at a population inversion of 0.7 , the values $W_{e} \tau_{\mathrm{rad}}=1$ and $W_{e} \tau_{\mathrm{rad}}=10$ yield upconversion rates of 46 and $120 \mathrm{~s}^{-1}$, respectively, which reduce the assumed radiative life time of $\tau_{\mathrm{rad}}=10 \mathrm{~ms}$ to effective lifetimes $\tau_{\text {eff }}=\left(\tau_{\text {rad }}^{-1}+W_{\mathrm{uc}}\right)^{-1}$ of 6.8 and $4.5 \mathrm{~ms}$, respectively.

Fig. 6 compares upconversion rates (still static upconversion) for the $\mathrm{Er}^{3+}$ concentrations $1.0 \cdot 10^{24} \mathrm{~m}^{-3}, 1.0$. $10^{25} \mathrm{~m}^{-3}$, and $1.0 \cdot 10^{26} \mathrm{~m}^{-3}$ in the case $W_{e} \tau_{\mathrm{rad}}=1$. The shape of the curves is seen not to change with the $\mathrm{Er}^{3+}$ concentration, and for $n_{2} \ll n_{\mathrm{Er}}$, the curves all coincide with the straight line representing the expression (22). However, although only excited $\mathrm{Er}^{3+}$ ions take part in energy-transfer processes (when migration is neglected), the rate of static upconversion not only depends on the excited state population concentration $n_{2}$, but also on the total $\mathrm{Er}^{3+}$ concentration $n_{\mathrm{Er}}$. This is explained as follows: for a given $n_{2}$, increasing $n_{\text {Er }}$ will increase the number of available sites for placing the $n_{2}$ excitations per volume unit. This increased freedom in combination with the negative correlation effect will lead

\footnotetext{
${ }^{5}$ In all simulations, the radiative lifetime of the ${ }^{4} I_{13 / 2}$ manifold is taken to be $\tau_{\text {rad }}=10 \mathrm{~ms}$. By inspection of (3), (7), and (8), it can be seen that when $R_{\mathrm{uc}}$ and $R_{\mathrm{mig}}$ are given, the upconversion rate is inversely proportional to $\tau_{\text {rad }}$, but that the value of $\tau_{\text {rad }}$ does not affect the shape of the $W_{\mathrm{uc}}$ curves.
} 


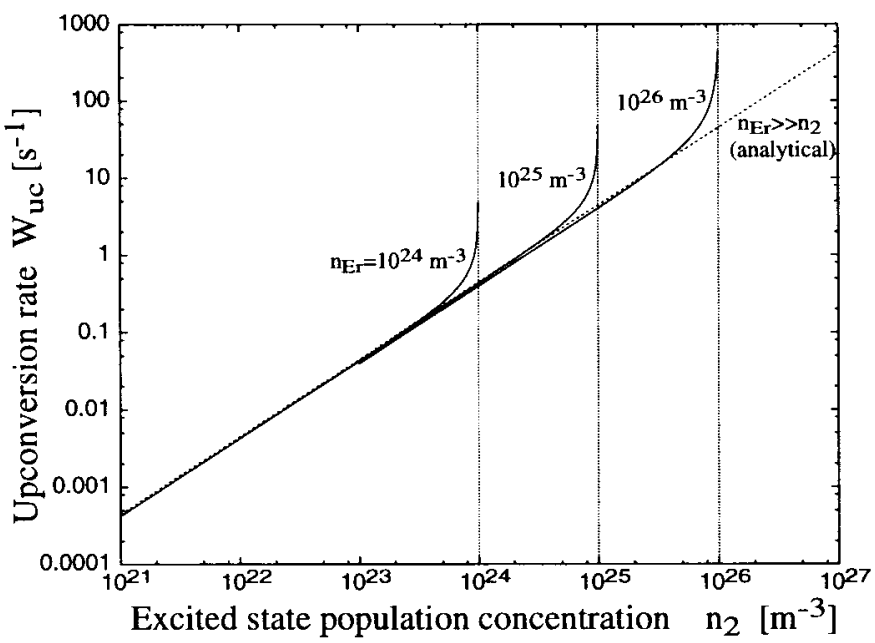

Fig. 6. Upconversion rate versus excited state population concentration for static upconversion for different values of the $\mathrm{Er}^{3+}$ concentration $n_{\mathrm{Er}}$. Other parameter values are $R_{\mathrm{uc}}=1.0 \mathrm{~nm}, R_{\max }=2 R_{\mathrm{uc}}$, and $W_{e}=1 / \tau_{\mathrm{rad}}=1 / 10 \mathrm{~ms}$. The curves represent averages over 100 systems, each containing $1000 \mathrm{Er}^{3+}$ ions.

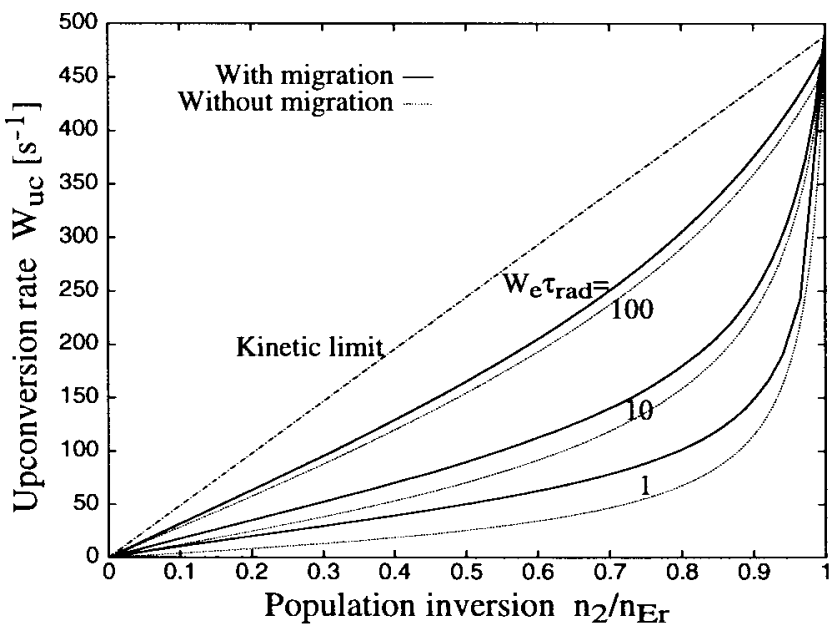

Fig. 7. Comparison of upconversion rates for static, respectively, migration-accelerated upconversion at an $\mathrm{Er}^{3+}$ concentration of $n_{\mathrm{Er}}=1.0$. $10^{26} \mathrm{~m}^{-3}$ for different values of the total emission rate $W_{e}$. Other parameter values are $R_{\mathrm{uc}}=1.0 \mathrm{~nm}, R_{\mathrm{mig}}=1.8 \mathrm{~nm}, R_{\max }=2 R_{\mathrm{mig}}$, and $\tau_{\mathrm{rad}}=$ $10 \mathrm{~ms}$. The curves represent averages over 10 systems, each containing 500 $\mathrm{Er}^{3+}$ ions.

to a spatial distribution of excited $\mathrm{Er}^{3+}$ ions that reduces the upconversion rate.

Next, we consider the influence of migration. For an $\mathrm{Er}^{3+}$ concentration of $n_{\mathrm{Fr}}=1.0 \cdot 10^{26} \mathrm{~m}^{-3}$, Fig. 7 compares upconversion rates for the cases with and without migration. As expected, migration increases the upconversion rate, because the diffusion of excitational energy will tend to even out differences in excitation probabilities and remove the negative correlation between the $\mathrm{Er}^{3+}$ ions. However, Fig. 7 indicates that the accelerating effect of migration is not sufficiently strong for bringing the system of $\mathrm{Er}^{3+}$ ions into the kinetic limit. Therefore, also in the case of migrationaccelerated upconversion, the total emission rate $W_{e}$ has great importance.

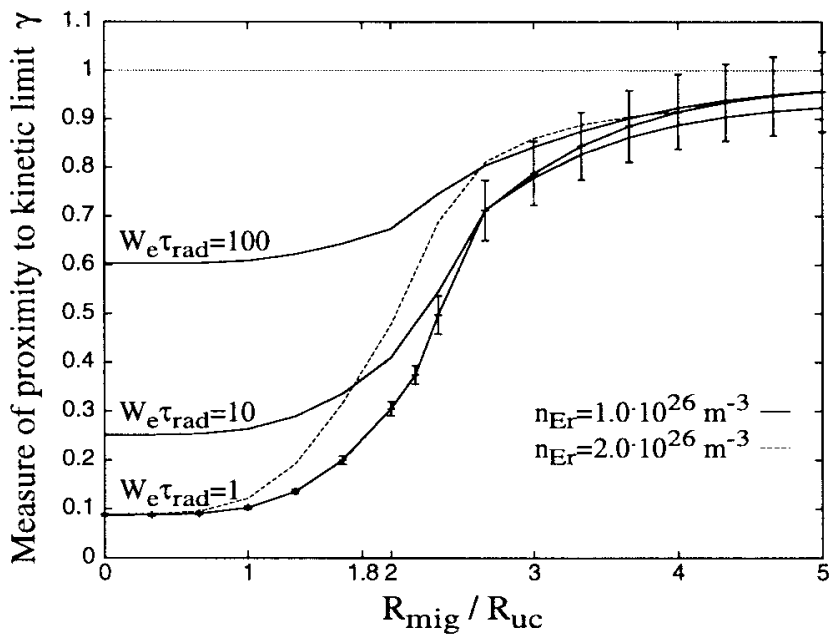

Fig. 8. Transition from static upconversion to the kinetic limit via migration-accelerated upconversion. For an $\mathrm{Er}^{3+}$ concentration of $n_{\mathrm{Er}}=$ $1.0 \cdot 10^{26} \mathrm{~m}^{-3}$, curves are shown for three different values of the total emission rate $W_{e}$. For the $\mathrm{Er}^{3+}$ concentration $n_{\mathrm{Er}}=2.0 \cdot 10^{26} \mathrm{~m}^{-3}$, only the $W_{e} \tau_{\text {rad }}=1$ curve is shown. Other parameter values are $R_{\mathrm{uc}}=$ $1.0 \mathrm{~nm}, R_{\max }=\max \left\{2 R_{\mathrm{mig}}, 3 R_{\mathrm{uc}}\right\}$, and $\tau_{\mathrm{rad}}=10 \mathrm{~ms}$. The curves represent averages over 10 systems, each containing $500 \mathrm{Er}^{3+}$ ions. The statistical uncertainties (standard deviations) on the shown simulation results are indicated by errorbars on the $\left(n_{\mathrm{Er}}=1.0 \cdot 10^{26} \mathrm{~m}^{-3}, W_{e} \tau_{\mathrm{rad}}=1\right)$ curve.

In order to examine how these conclusions depend on the $\mathrm{Er}^{3+}$ concentration and on the strength of the migration process, we introduce the quantity

$$
\gamma=\frac{C_{\mathbf{u c}}^{(0)}}{C_{\mathbf{u c}}^{\text {kin }}}=\frac{\left.\frac{d W_{\mathbf{u c}}}{d n_{2}}\right|_{n_{2}=0}}{\frac{d W_{\mathbf{u c}}^{\text {kin }}}{d n_{2}}}
$$

as a measure of how close the conditions are to the kinetic limit (with $\gamma=1$ corresponding to the kinetic limit). Fig. 8 shows $\gamma$ as a function of the ratio $R_{\mathrm{mig}} / R_{\mathrm{uc}}$ of the strength parameters of migration and upconversion.

As expected, $\gamma$ increases and tends toward 1 with increasing $R_{\mathrm{mig}} / R_{\mathrm{uc}}$, i.e., the kinetic limit would be reached in a host material, in which the migration process between $\mathrm{Er}^{3+}$ ions was sufficiently strong. Furthermore, Fig. 8 shows that $\gamma$ increases with $W_{e}$, because-as earlier mentioned-the exchange of excited ions has an effect similar to migration. Finally, $\gamma$ is seen to grow with the $\mathrm{Er}^{3+}$ concentration. Thus, in contrast to the case of static upconversion, the shape of the $W_{\mathrm{uc}}$ curves will change with the $\mathrm{Er}^{3+}$ concentration, since the conditions approach the kinetic limit, when the $\mathrm{Er}^{3+}$ concentration is increased. However, at the $\mathrm{Er}^{3+}$ concentrations considered in Fig. 8, a doubling of the ratio $R_{\mathrm{mig}} / R_{\mathrm{uc}}$ (compared to the assumed value of 1.8) is needed to reach the kinetic limit. This corresponds to a multiplication of the ratio $P_{\mathrm{mig}} / P_{\mathrm{uc}}$ by a factor of $2^{6}=64$. Such a span in parameter values seems unlikely within silica glasses (the value 1.8 came from measurements on silica glasses, cf. Section II). We thus conclude that in silica glasses with $\mathrm{Er}^{3+}$ concentrations of $1.0-2.0 \cdot 10^{26} \mathrm{~m}^{-3}$ or below, the kinetic limit is not reached, and consequently a quadratic model $g_{\mathbf{u c}}=W_{\mathbf{u c}} n_{2}=$ 
$C_{\text {uc }} n_{2}^{2}$ is generally not adequate ${ }^{6}$ for describing homogeneous upconversion in such glasses. Instead, we suggest a different explanation for the experimental observations of quadratic upconversion [4], [23]. From Figs. 5 and 7, it is seen that the $W_{\mathbf{u c}}$ curves do not deviate strongly from their tangents at 0 until the population inversion reaches values around 0.7 . In other words, the quadratic upconversion model $g_{\mathbf{u c}}=$ $C_{\mathrm{uc}}^{(0)} n_{2}^{2}$, which in Section III was shown to be valid at low population inversions, with good approximation holds in the range of population inversions from 0 to $0.6-0.7$. We propose this phenomenon rather than the kinetic limit as the explanation for the experimental observations of quadratic upconversion.

In [4], the number of upconversion processes versus excited state population is measured by pumping $\mathrm{Er}^{3+}$-doped fibers at $1480 \mathrm{~nm}$ and by detecting flourescence around $1500 \mathrm{~nm}$ (proportional to $n_{2}$ ) and around $980 \mathrm{~nm}$ (proportional to $g_{\mathbf{u c}}$ ). Because of pump-stimulated emission, the method does not allow investigations at population inversions above c. 0.75 (the actual value depends on the codopants). The results in [4] show a quadratic dependence of $g_{\mathbf{u c}}$ on $n_{2}$ at low population inversions, i.e., a straight line in a double-logarithmic $n_{2}-g_{\mathbf{u c}}$ coordinate system, followed by an upwards bending of the $g_{\text {uc }}$ curve at higher population inversions. This basic pattern is in accordance with our simulation results. However, in [4], a quadratic model for homogeneous upconversion is assumed, and the upwards bending of the curve is attributed to the presence of pairs of $\mathrm{Er}^{3+}$ ions. Indeed, the presence of pairs or larger clusters of $\mathrm{Er}^{3+}$ ions will give rise to an upwards bending of the upconversion curve, but so will also homogeneous upconversion, according to the simulation results presented in this paper. ${ }^{7}$ Thus, the simulation results could have important implications for the interpretation of experimental results, when it comes to separating the upconversion contributions of homogeneously and clustered $\mathrm{Er}^{3+}$ ions with the purpose of estimating the fraction of clustered ions. Furthermore, for erbium-doped waveguiding structures in which homogeneous upconversion plays a significant role, the simulation results can be expected to have importance for the design of amplifying components. Thus, according to the above presented simulation results, a further increase of the population inversion above 0.7 (with the purpose of improving gain and noise figure) will be more expensive (in terms of extra pump power) than predicted by a purely quadratic upconversion model (fitted at low population inversions). This is a consequence of the strongly growing slope of the $W_{\mathbf{u c}}$ curves in Fig. 7.

\section{CONCLUSION}

It has been demonstrated that the treatment of energytransfer upconversion between $\mathrm{Er}^{3+}$ ions requires the inclusion

\footnotetext{
${ }^{6}$ Nor do the results give any indication that a cubic model $g_{\mathrm{uc}}=W_{\mathrm{uc}} n_{2}=$ $\tilde{C}_{\mathrm{uc}} n_{2}^{3}$ should be valid.

${ }^{7}$ One might object that the upwards bending of the homogeneous upconversion curves is due to the fact that with a random ion distribution, a small fraction of the $\mathrm{Er}^{3+}$ ions will have neighboring $\mathrm{Er}^{3+}$ ions within a distance so short that it corresponds to the presence of pairs. This argument is, however, only partly true, since the negative correlation effect will give rise to an upwards bending of the upconversion curves for any ion distribution, even for a (hypothetic) regular lattice of equidistantly placed $\mathrm{Er}^{3+}$ ions.
}

of the negative correlation effect, and therefore the classical quenching theory is not suitable for this purpose. A method of describing the behavior of interacting $\mathrm{Er}^{3+}$ ions has been developed, based on a system of differential equations for the excitation probabilities of the ions. This has been combined with Monte Carlo simulations of the ion positions in order to perform calculations of the quenching rate due to upconversion.

Upconversion alters the spatial distribution of $\mathrm{Er}^{3+}$ ions excited to the ${ }^{4} I_{13 / 2}$ manifold, not only by creating differences in single-ion excitation probabilities, but also by introducing negative correlation between the $\mathrm{Er}^{3+}$ ions. These effects reduce the extent of upconversion compared to a random distribution of excited $\mathrm{Er}^{3+}$ ions. Two physical mechanisms, migration of excitational energy and the exchange of excited ions by emission and absorption, have the opposite effect, i.e., they bring the distribution of excited $\mathrm{Er}^{3+}$ ions closer to a random distribution.

Contrary to what is frequently assumed in the literature, our simulations indicate that for silica glasses with $\mathrm{Er}^{3+}$ concentrations of $1.0-2.0 \cdot 10^{26} \mathrm{~m}^{-3}$ or below, migration is not strong enough for the kinetic limit to be reached. Therefore, upconversion does not in general depend quadratically on the population inversion. Instead, experimental observations of quadratic upconversion may be explained as a phenomenon at low population inversions (below $0.6-0.7)$.

The impact of the simulation results on the interpretation of experiments and on the design of high-concentration erbiumdoped devices has been briefly discussed, but should be the object of future work.

\section{APPENDIX \\ DERIVATION OF DIFFERENTIAL EQUATIONS FOR THE PROBABILITIES $p_{k}$ AND $p_{k \wedge l}$}

First, we consider the probability $p_{k}(t)$ of finding the $k$ th $\mathrm{Er}^{3+}$ ion excited to the ${ }^{4} I_{13 / 2}$ manifold at time $t$. Contributions to changes in $p_{k}(t)$ come from emission, absorption, upconversion, and migration. Emission and absorption contribute to the time derivative of $p_{k}$ with terms $-W_{e} p_{k}$ and $W_{a}\left(1-p_{k}\right)$, respectively. The probability per time unit for an upconversion proces with ion $k$ as the donor and ion $l$ as the acceptor is $1 / 2 P_{\mathbf{u c}}\left(\left|\boldsymbol{r}_{k}-\boldsymbol{r}_{l}\right|\right) p_{k \wedge l}$. The probability of migration from $l$ to $k$ is $P_{\text {mig }}\left(\left|\boldsymbol{r}_{k}-\boldsymbol{r}_{l}\right|\right) p_{\neg k \wedge l}$, where $p_{\neg k \wedge l}$ denotes the probability of finding $l$ excited and $k$ unexcited. Analogously, the probability of migration from $k$ to $l$ equals $P_{\operatorname{mig}}\left(\left|\boldsymbol{r}_{k}-\boldsymbol{r}_{l}\right|\right) p_{k \wedge \neg l}$. All terms corresponding to energytransfer processes should be summed with respect to $l$. This leads to the equation

$$
\begin{aligned}
\frac{d p_{k}}{d t}= & -W_{e} p_{k}+W_{a}\left(1-p_{k}\right)-\sum_{l \neq k} \frac{1}{2} P_{\mathbf{u c}}\left(\left|\boldsymbol{r}_{k}-\boldsymbol{r}_{l}\right|\right) p_{k \wedge l} \\
& +\sum_{l \neq k} P_{\mathrm{mig}}\left(\left|\boldsymbol{r}_{k}-\boldsymbol{r}_{l}\right|\right) p_{\neg k \wedge l} \\
& -\sum_{l \neq k} P_{\mathrm{mig}}\left(\left|\boldsymbol{r}_{k}-\boldsymbol{r}_{l}\right|\right) p_{k \wedge \neg l}
\end{aligned}
$$




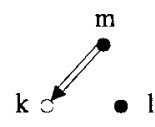

A

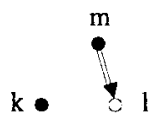

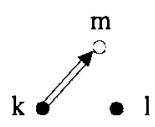

$\mathrm{C}$

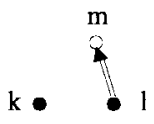

$\mathrm{D}$
Fig. 9. The four situations in which migration among the $\mathrm{Er}^{3+}$ ions $k, l$, and $m$ contributes to the time derivative of $p_{k \wedge l}$. Excited ions are represented by full circles.

By inserting $p_{\neg k \wedge l}-p_{k \wedge \neg l}=p_{l}-p_{k}$, (27) can be rewritten as

$$
\begin{aligned}
\frac{d p_{k}}{d t}= & \sum_{l \neq k}\left[P_{\operatorname{mig}}\left(\left|\boldsymbol{r}_{k}-\boldsymbol{r}_{l}\right|\right)\left(p_{l}-p_{k}\right)\right. \\
& \left.-\frac{1}{2} P_{\mathbf{u c}}\left(\left|\boldsymbol{r}_{k}-\boldsymbol{r}_{l}\right|\right) p_{k \wedge l}\right] \\
& -W_{e} p_{k}+W_{a}\left(1-p_{k}\right)
\end{aligned}
$$

identical to (7).

Next, we consider the joint probability $p_{k \wedge l}(t)$ of finding the $\mathrm{Er}^{3+}$ ions $k$ and $l$ both excited to the ${ }^{4} I_{13 / 2}$ manifold. Contributions to the time derivative of $p_{k \wedge l}$ only come from processes that change the validity of the assertion that ions $k$ and $l$ are both excited. Thus, emission gives rise to a term $-2 W_{e} p_{k \wedge l}$, and absorption is represented by a term $W_{a}\left(p_{k}+p_{l}-2 p_{k \wedge l}\right)$, where $p_{k}+p_{l}-2 p_{k \wedge l}$ is the probability of finding exactly one of the ions $k$ and $l$ excited. Upconversion between the ions $k$ and $l$ yields a term $-P_{\mathbf{u c}}\left(\left|\boldsymbol{r}_{k}-\boldsymbol{r}_{l}\right|\right) p_{k \wedge l}$, whereas upconversion between $k$ and a third ion $m$ gives a term $-\frac{1}{2} P_{\mathbf{u c}}\left(\left|\boldsymbol{r}_{k}-\boldsymbol{r}_{m}\right|\right) p_{k \wedge l \wedge m}$, since this process only contributes to the time derivative of $p_{k \wedge l}$, if $l$ is also excited. Upconversion between $l$ and $m$ is treated similarly.

Migration among the three $\mathrm{Er}^{3+}$ ions $k, l$, and $m$ can occur in 12 different situations, when the choices of donor ion, acceptor ion, and excitational state of the third ion are taken into consideration. Of these 12 situations, only the four shown in Fig. 9 contribute to the time derivative of $p_{k \wedge l}$.

The respective contributions are

$$
\begin{array}{ll}
\text { A: } & P_{\mathrm{mig}}\left(\left|\boldsymbol{r}_{k}-\boldsymbol{r}_{m}\right|\right) p_{\neg k \wedge l \wedge m} \\
\mathrm{~B}: & P_{\mathrm{mig}}\left(\left|\boldsymbol{r}_{l}-\boldsymbol{r}_{m}\right|\right) p_{k \wedge \neg l \wedge m} \\
\mathrm{C}: & -P_{\mathrm{mig}}\left(\left|\boldsymbol{r}_{k}-\boldsymbol{r}_{m}\right|\right) p_{k \wedge l \wedge \neg m} \\
\mathrm{D}: & -P_{\mathrm{mig}}\left(\left|\boldsymbol{r}_{l}-\boldsymbol{r}_{m}\right|\right) p_{k \wedge l \wedge \neg m} .
\end{array}
$$

These terms can be joined two by two by using

$$
\begin{aligned}
& p_{\neg k \wedge l \wedge m}-p_{k \wedge l \wedge \neg m}=p_{l \wedge m}-p_{k \wedge l} \\
& p_{k \wedge \neg l \wedge m}-p_{k \wedge l \wedge \neg m}=p_{k \wedge m}-p_{k \wedge l}
\end{aligned}
$$

and the equation for $p_{k \wedge l}$ then becomes

$$
\begin{aligned}
\frac{d p_{k \wedge l}}{d t}=\sum_{m \neq k, m \neq l} & {\left[P_{\text {mig }}\left(\left|\boldsymbol{r}_{k}-\boldsymbol{r}_{m}\right|\right)\left(p_{l \wedge m}-p_{k \wedge l}\right)\right.} \\
& -\frac{1}{2} P_{\mathbf{u c}}\left(\left|\boldsymbol{r}_{k}-\boldsymbol{r}_{m}\right|\right) p_{k \wedge l \wedge m} \\
& +P_{\text {mig }}\left(\left|\boldsymbol{r}_{l}-\boldsymbol{r}_{m}\right|\right)\left(p_{k \wedge m}-p_{k \wedge l}\right) \\
& \left.-\frac{1}{2} P_{\mathbf{u c}}\left(\left|\boldsymbol{r}_{l}-\boldsymbol{r}_{m}\right|\right) p_{k \wedge l \wedge m}\right] \\
& -2 W_{e} p_{k \wedge l}+W_{a}\left(p_{k}+p_{l}-2 p_{k \wedge l}\right) \\
& -P_{\mathbf{u c}}\left(\left|\boldsymbol{r}_{k}-\boldsymbol{r}_{l}\right|\right) p_{k \wedge l}
\end{aligned}
$$

identical to (8).

\section{ACKNOWLEDGMENT}

The authors thank J. Nilsson and M. Hempstead, University of Southampton, together with T. Feuchter and M. Kristensen from Mikroelektronikcentret, J. Engholm Pedersen, NKT Research Center, and M. S. Berger, C. Lester, and J. H. Povlsen from the Department of Electromagnetic Systems for useful discussions.

\section{REFERENCES}

[1] M. Shimizu, M. Yamada, M. Horiguchi, and E. Sugita, "Concentration effect on optical amplification characteristics of Er-doped silica singlemode fibers," IEEE Photon. Technol. Lett., vol. 2, pp. 43-45, 1990.

[2] O. Lumholt, A. Bjarklev, T. Rasmussen, and C. Lester, "Rare earthdoped integrated glass components: Modeling and optimization," $J$. Lightwave Technol., vol. 13, pp. 275-282, 1995.

[3] F. Di Pasquale, M. Zoboli, M. Federighi, and I. Massarek, "Finiteelement modeling of silica waveguide amplifiers with high erbium concentration," IEEE J. Quantum Electron., vol. 30, pp. 1277-1282, 1994.

[4] J. Nilsson, P. Blixt, B. Jaskorzynska, and J. Babonas, "Evaluation of parasitic upconversion mechanisms in $\mathrm{Er}^{3+}$-doped silica-glass fibers by analysis of flourescence at $980 \mathrm{~nm}$, , J. Lightwave Technol., vol. 13, pp. 341-349, 1995

[5] M. Hempstead, J. E. Román, C. Ye, J. S. Wilkinson, P. Camy, P. Laborde, and C. Lerminiaux, "Anomalously high uniform upconversion in an erbium-doped waveguide amplifier," in Proc. 7th Eur. Conf. Integrated Optics (ECIO'95), 1995, pp. 233-236, Paper TuC4.

[6] E. Delevaque, T. Georges, M. Monerie, P. Lamouler, and J.-F. Bayon, "Modeling of pair-induced quenching in erbium-doped silicate fibers," IEEE Photon. Technol. Lett., vol. 5, pp. 73-75, 1993.

[7] P. F. Wysocki, J. L. Wagener, M. J. F. Digonnet, and H. J. Shaw, "Evidence and modeling of paired ions and other loss mechanisms in erbium-doped silica fibers," SPIE, Fiber Laser Sources and Amplifiers IV, vol. 1789 pp. 66-79, 1992.

[8] M. K. Davis, M. J. F. Digonnet, and R. H. Pantell, "Characterization of clusters in rare earth-doped fibers by transmission measurements," $J$. Lightwave Technol., vol. 13, pp. 120-126, 1995.

[9] J. L. Wagener, P. F. Wysocki, M. J. F. Digonnet, H. J. Shaw, and D J. DiGiovanni, "Effects of concentration and clusters in erbium-doped fiber lasers," Opt. Lett., vol. 18, no. 23, pp. 2014-2016, 1993.

[10] W. J. Miniscalco, "Erbium-doped glasses for fiber amplifiers at 1500 nm," J. Lightwave Technol., vol. 9, pp. 234-250, 1991.

[11] B. J. Ainslie, S. P. Craig-Ryan, S. T. Davey, J. R. Armitage, C. G Atkins, and R. Wyatt, "Optical analysis of erbium doped fibers for efficient lasers and amplifiers," in Proc. IOOC'89, 1989, pp. 22-23, Paper 20A3-2.

[12] M. J. F. Digonnet, M. K. Davis, and R. H. Pantell, "Rate equations for clusters in rare earth-doped fibers," Opt. Fiber Technol., vol. 1, pp. 48-58, 1994.

[13] E. Desurvire, Erbium Doped Amplifiers: Principals and Applications, 1st ed. New York: Wiley, 1994.

[14] R. S. Quimby, W. J. Miniscalco, and B. Thompson, "Clustering in erbium-doped silica glass fibers analyzed using $980 \mathrm{~nm}$ excited-state absorption," J. Appl. Phys., vol. 76, no. 8, pp. 4472-4478, 1994.

[15] A. Bjarklev, Optical Fiber Amplifiers: Design and System Applications, 1st ed. Boston, MA: Artech House, 1993.

[16] W. J. Miniscalco, "Optical and electronic properties of rare earth ions in glasses," in Rare Earth Doped Fiber Lasers and Amplifiers, Michel J. F. Digonnet, Ed., 1st ed. New York: Marcel Dekker, 1993, ch. 2

[17] D. L. Dexter, "A theory of sensitized luminescence in solids," J. Chem. Phys., vol. 21, no. 5, pp. 836-850, 1953.

[18] J. E. Román, C. Ye, and M. Hempstead, " $1.7 \mu$ m excited state absorption measurement in erbium-doped silicate glasses," in Integrated Photonics Research, OSA Tech. Dig. Ser. Washington DC: Opt. Soc. Amer., 1995, vol. 7, pp. 221-223, Paper IFC4.

[19] K. B. Eisenthal and S. Siegel, "Influence of resonance transfer on luminescence decay," J. Chem. Phys., vol. 41, no. 3, pp. 652-655, 1964.

[20] A. I. Bŭrshtein, "Concentration quenching of noncoherent excitation in solutions," Sov. Phys. Uspekhi, vol. 27, no. 8, pp. 579-606, 1984.

[21] V. P. Gapontsev and N. S. Platonov, "Migration-accelerated quenching of luminescence in glasses activated by rare-earth ions," in Dynamical Processes in Disordered Systems, W. M. Yen, Ed., 1st ed., vol. 50 of Materials Science Forum. Aedermannsdorf, Switzerland: Trans Tech Pub., 1989, pp. 165-222. 
[22] W. Q. Shi, M. Bass, and M. Birnbaum, "Effects of energy-transfer among $\mathrm{Er}^{3+}$ ions on the flourescence decay and lasing properties of heavily doped Er: $\mathrm{Y}_{3} \mathrm{Al}_{5} \mathrm{O}_{12}$." J. Opt. Soc. Amer. B, vol. 7, no. 8, pp. 1456-1462, 1990.

[23] R. Wyatt, "Spectroscopy of rare earth doped fibers," SPIE, Fiber Laser Sources and Amplifiers, vol. 1171, pp. 54-64, 1989.

[24] B. Pálsdóttir and C. C. Larsen, "Dipole-dipole model for upconversion and flourescence lifetime in erbium doped fibers," in Optical Amplifiers and Their Applications, Proc. OAA'94 Tech. Dig. Ser., 1994, vol. 14, pp. 83-85, Paper ThB5.

[25] W. J. C. Grant, "Role of rate equations in the theory of luminescent energy transfer," Phys. Rev. B, vol. 4, no. 2, pp. 648-663, 1971.

[26] J. C. Wright, "Up-conversion and excited state energy transfer in rareearth doped materials," in Radiationless Processes in Molecules and Condensed Phases, F. K. Fong, Ed., vol. 15 of Topics in Applied Physics, 1st ed. Berlin, Germany: Springer-Verlag, 1976, pp. 239-295.

[27] C. Y. Chen, R. R. Petrin, D. C. Yeh, and W. A. Sibley, "Concentrationdependent energy-transfer processes in $\mathrm{Er}^{3+}$ - and $\mathrm{Tm}^{3+}$-doped heavymetal flouride glass", Opt. Lett., vol. 14, no. 9, pp. 432-434, 1989.

[28] B. Pedersen, M. L. Dakss, B. A. Thompson, W. J. Miniscalco, T. Wei, and L. J. Andrews, "Experimental and theoretical analysis of efficient erbium-doped fiber power amplifiers," IEEE Photon. Technol. Lett., vol. 3, pp. 1085-1087, 1991.

[29] K. Hattori, T. Kitagawa, M. Oguma, Y. Ohmori, and M. Horiguchi, "Erbium-doped silica-based waveguide amplifier integrated with a 980/1530 nm wdm coupler," Electron. Lett., vol. 30, no. 11, pp. 856-857, 1994.

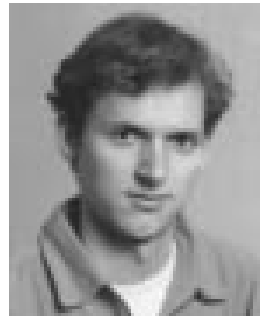

Jacob L. Philipsen was born in Copenhagen, Denmark, on November 22, 1972. He received the M.Sc. degree in engineering in 1995 from the Electromagnetics Institute (now Department of Electromagnetic Systems), Technical University of Denmark, Lyngby, Denmark. He is currently pursuing the Ph.D. degree at the Center for Broadband Telecommunications, Department of Electromagnetic Systems, Technical University of Denmark, focusing on rareearth-doped integrated optics.

Anders Bjarklev (M'92) was born in Roskilde, Denmark, on July 2, 1961. $\mathrm{He}$ received the M.Sc. degree in electrical engineering and the Ph.D. degree in 1985 and 1988, respectively, from the Technical University of Denmark, Lyngby, Denmark, and the Dr.Technices degree in 1995.

His research interests are primarily within the field of optical waveguides and electromagnetic theory. Presently, he is employed as Associate Professor at the Department of Electromagnetic Systems (formerly Electromagnetics Institute), Technical University of Denmark. He is the author of the book Optical Amplifiers: Design and System Applications (Boston, MA: Artech House, 1993). 